\title{
A Case with Neutropenia Related with the Use of Various Atypical Antipsychotics
}

\author{
Myung Ho Lim¹, Jong-II Park², and Tae Won Park ${ }^{2,3} \bowtie$ \\ ${ }^{1}$ Department of Psychiatry, College of Medicine, Dankook University, Cheonan, Republic of Korea \\ ${ }^{2}$ Department of Psychiatry, Chonbuk National University Medical School, Jeonju, Republic of Korea \\ ${ }^{3}$ Research Institute of Clinical Medicine of Chonbuk National University-Biomedical Research Institute of Chonbuk National University Hospital, \\ Jeonju, Republic of Korea
}

Herein, we report here a case of a 21 -year-old patient with a conduct disorder, who had neutropenia associated with treatment with 4 different antipsychotics (olanzapine, quetiapine, risperidone, and aripiprazole) on a sequential basis. This case supports the hypothesis that patients who developed antipsychotic-induced neutropenia on one medication are more likely to develop neutropenia when taking other antipsychotics. Based on this finding, we may suggest that the number of white blood cell and neutrophil counts in patients with a history of antipsychotic-induced neutropenia needs to be carefully monitored during antipsychotic treatment.

Psychiatry Investig 2013;10:428-431

Key Words Multiple atypical antipsychotics, Neutropenia.

\section{INTRODUCTION}

Previous reports have described that clozapine, a second generation antipsychotics, carries the highest risk of neutropenia and agranulocytosis among adults in Western countries (approximately $3 \%$ and $0.8 \%$, respectively). ${ }^{1}$ However, neutropenia has also been reported when patients were treated with other second generation antipsychotics such as olanzapine ${ }^{2}$ quetiapine,${ }^{3}$ risperidone, ${ }^{4}$ and amisulpride. ${ }^{5}$ Although the risk of these individual medicines has been documented, it is still not clear whether the risk of neutropenia is further increased when a patient is sequentially treated with different antipsychotics. Herein, we report a case of drug-induced neutropenia associated with 4 different antipsychotics (olanzapine, quetiapine, risperidone, and aripiprazole) that demonstrates an increased susceptibility to neutropenia after the initial olanzapine-induced neutropenic event. To our knowledge, this report is the first to involve 4 different antipsycho-

Received: February 9, 2013 Revised: February 13, 2013

Accepted: February 13, 2013 Available online: December 16, 2013

$\triangle$ Correspondence: Tae Won Park, MD, PhD

Department of Psychiatry, Chonbuk National University Medical School, 63418 Geumam-dong, Jeonju 561-712, Republic of Korea

Tel: +82-63-250-2028, Fax: +82-63-275-3157, E-mail: ptaewon@jbnu.ac.kr

(c) This is an Open Access article distributed under the terms of the Creative Commons Attribution Non-Commercial License (http://creativecommons.org/licenses/bync/3.0) which permits unrestricted non-commercial use, distribution, and reproduction in any medium, provided the original work is properly cited. tic medications in the development of neutropenia.

\section{CASE}

A 21-year-old Asian adolescent with a conduct disorder started treatment in 2006. We prescribed him risperidone 2 $\mathrm{mg}$ and valoproic acid $750 \mathrm{mg}$. The patient was maintained on this regimen for a year; his white blood cell (WBC) and neutrophil counts were $6.38 \times 10^{9} / \mathrm{L}$ and $3.04 \times 10^{9} / \mathrm{L}$, respectively, in March 2007 (Figure 1). In 2009, the patient was admitted a second time for aggression, refusal to stay in school, and unstable mood. We maintained an outpatient regimen that included olanzapine $10 \mathrm{mg}$ and valproate $1000 \mathrm{mg}$, which had been changed 3 months before hospitalization. At the time of hospitalization, WBC and neutrophil counts were $4.56 \times 10^{9} / \mathrm{L}$ and $1.49 \times 10^{9} / \mathrm{L}$, respectively. During the hospitalization, repeated neutrophil counts were examined, and they were $1.69 \times 10^{9} / \mathrm{L}, 1.84 \times 10^{9} / \mathrm{L}$, and $1.47 \times 10^{9} / \mathrm{L}$. Two months after the hospitalization, he was discharged with residual symptoms of verbal aggression and internet addiction. In March 2010, we changed the patient's medications olanzapine to quetiapine based on the complaints of weight gain and sleep disturbances; subsequently, we increased his dose to $800 \mathrm{mg}$ in combination with $1000 \mathrm{mg}$ of valproic acid. During this period, we examined WBC and neutrophil count only once a year because the patient was not neutropenic. In January 


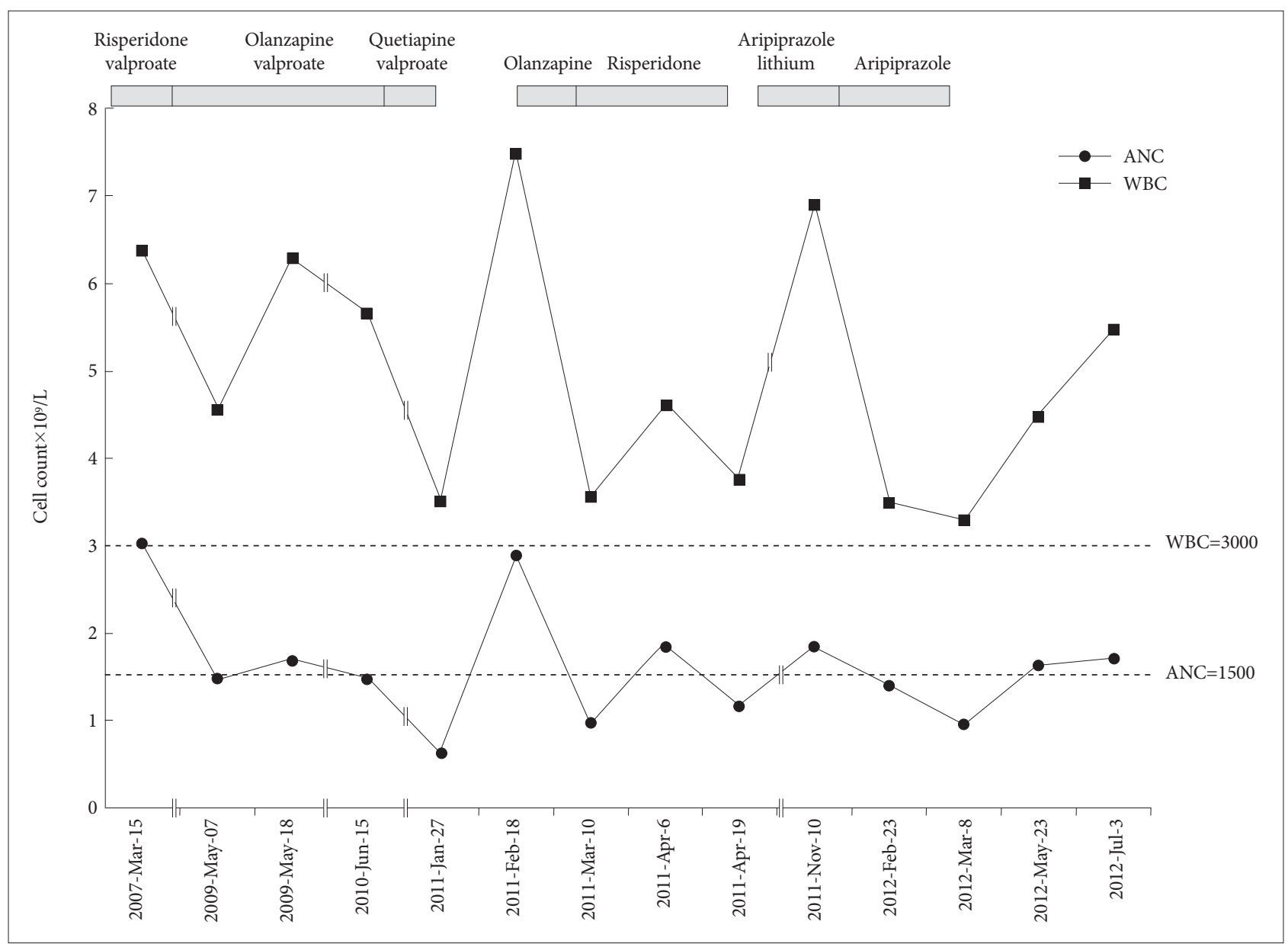

Figure 1. Follow up WBC and neutrophil counts during the treatment. WBC: white blood cells, ANC: absolute neutrophil count.

2011, the patient's WBC and neutrophil counts had dropped to $3.51 \times 10^{9} / \mathrm{L}$ and $0.63 \times 10^{9} / \mathrm{L}$, respectively; therefore, all medications were discontinued. There was no evidence of infection at that time. He received a diagnosis of drug-induced neutropenia based on the hematologic examination, which includes a negative finding of fluorescent antinuclear antibody (FANA) test and PB smear. Three weeks later, WBC and neutrophil counts returned to normal as they were $7.48 \times 10^{9} / \mathrm{L}$ and $2.9 \times 10^{9} / \mathrm{L}$, respectively. Then, we restarted the patient on olanzapine $5 \mathrm{mg}$ because this medication had demonstrated effectiveness towards treatment of the patient's aggression. Three weeks after the medication, WBC and neutrophil counts had dropped again to $3.56 \times 10^{9} / \mathrm{L}$ and $0.98 \times 10^{9} / \mathrm{L}$, respectively. Therefore, we ceased olanzapine and changed to risperidone up to $2 \mathrm{mg}$, which he had previously been tolerated without hematologic side effects. Two weeks after the initiation of the new regimen, WBC and neutrophil counts were increased to $4.61 \times 10^{9} / \mathrm{L}$ and $1.85 \times 10^{9} / \mathrm{L}$, respectively, but 2 weeks later they dropped again to $3.76 \times 10^{9} / \mathrm{L}$ and $1.17 \times 10^{9} / \mathrm{L}$. Then, we discontinued risperidone and followed the patient without any medication. Since his symptoms per- sisted and the patient experienced emotional problems during his 5 month medication cessation, we tried aripiprazole 5 mg in September 2011 and added lithium $600 \mathrm{mg}$. Although the patient took these medicines irregularly, WBC and neutrophil counts were increased to $6.9 \times 10^{9} / \mathrm{L}$ and $1.85 \times 10^{9} / \mathrm{L}$, respectively. We maintained him with aripiprazole in combination with lithium for 5 months, but stopped lithium due to GI troubles in February 2012. At that point, WBC and neutrophil counts were $3.5 \times 10^{9} / \mathrm{L}$ and $1.4 \times 10^{9} / \mathrm{L}$, respectively. However, after 2 weeks of single medication treatment with aripiprazole, the patient's WBC and neutrophil numbers had dropped to $3.29 \times 10^{9} / \mathrm{L}$ and $0.97 \times 10^{9} / \mathrm{L}$ again. We ceased the patient's medication again and planned to follow him without his taking medication. At the follow-up visit, the patient's WBC and neutrophil counts had increased, returning to normal levels of $5.48 \times 10^{9} / \mathrm{L}, 1.72 \times 10^{9} / \mathrm{L}$, but were sustained at a lower level than before the initiation of the treatment with antipsychotic medication. This case report was approved by the Chonbuk National University Hospital Review Board. 


\section{DISCUSSION}

In this case, there was no history of use of any other hematologically toxic medication other than the prescribed antipsychotics, and no other cause of neutropenia was identified. At first, the possibility of valproate in combination with olanzapine as the causative agents was considered, given a case report demonstrating severe neutropenia caused by valproate. ${ }^{6}$ However, risperidone and valproate did not cause neutropenia when given to this patient.

It has been suggested that valproate is associated with a minimal, presumably not clinically significant, decrease in plasma olanzapine levels. ${ }^{7}$ In contrast, it is thought that valproate has been associated with increases of up to $77 \%$ in quetiapine plasma levels. ${ }^{8}$ It is possible that in this case, the level of quetiapine was unexpectedly high. However, there was no evidence of any other adverse effects of quetiapine. Since olanzapine and quetiapine are similar to clozapine in terms of chemical structure and pharmacological profile, a similar mechanism of antipsychotic-induced neutropenia can be proposed. ${ }^{1}$

Since risperidone and aripiprazole have different chemical structures compared to clozapine, olanzapine, and quetiapine, it appears that other mechanisms are involved. First of all, risperidone did not produce neutropenia at first exposure in our case. After the initial development of antipsychotic-induced neutropenia, however, rechallenging with risperidone began to induce a neutropenic state. There has been no report of neutropenia associated with aripiprazole as a single agent. In addition, some case reports suggested that aripiprazole can be used in the case of antipsychotic-induced neutropenia. ${ }^{5,9}$ However, it needs to be reconsidered because, similar with our case, aripiprazole-induced neutropenia subsequent to other antipsychotic-induced neutropenia were reported. ${ }^{10}$

There have been case reports of multi-antipsychotic-induced neutropenia. Typically, these involved single use of 2 different antipsychotics, clozapine and olanzapine, ${ }^{11}$ in addition to the combinations of risperidone and clozapine, ${ }^{12}$ risperidone and aripiprazole, ${ }^{9}$ and olanzapine and ziprasidone. ${ }^{13}$ Currently, there is one case report about neutropenia associated with the use of 3 different antipsychotics, quetiapine, olanzapine, and aripiprazole..$^{14}$ In agreement with this report, we found that the neutrophil count remarkably dropped to $0.63 \times 10^{9} / \mathrm{L}$ with quetiapine, $0.98 \times 10^{9} / \mathrm{L}$ with olanzapine, $1.17 \times 10^{9} / \mathrm{L}$ with risperidone, and $0.97 \times 10^{9} / \mathrm{L}$ with aripiprazole after the initial even. It is notable that the neutrophil count was $1.47 \times 10^{9} / \mathrm{L}$, which is slightly below than normal range when initial neutropenia developed due to olanzapine in our case. These cases may suggest that patients who previously suffered antipsychotic-induced neutropenia were likely to develop more severe neutropenia when taking other antipsychotics compared to the initial event.

It is interesting that $\mathrm{WBC}$ and neutrophil counts remained lower than before the treatment of antipsychotic drug until 4 months later, after the cessation of antipsychotic drug. Since findings of FANA and PB smear were negative, we hypothesized that long-term bone marrow suppression plays a role in repeated antipsychotic-induced neutropenia. However, little is known about the mechanism of antipsychotic-induced neutropenia. The mechanism of clozapine-induced neutropenia is thought to be the formation of a nitrenium cation catalyzed by the flavin-containing monooxygenase-3 (FMO3) system of leukocytes seen with both clozapine and olanzapine..$^{15-17} \mathrm{~A}$ recent report showed that inactivated clozapine may itself exert toxic effects. ${ }^{18}$ Although antipsychotic-induced neutropenia can result in serious complications, especially in combination with signs of infection or agranulocytosis, only clozapine has clinical guidelines for blood monitoring with the administration. For these reasons, we may suggest that careful monitoring of WBC counts in combination with other examinations to detect signs of possible infection is critical for patients with a history of antipsychotic-induced neutropenia.

\section{Acknowledgments}

The present research was conducted by the research fund of Dankook University in 2013.

\section{REFERENCES}

1. Nooijen PM, Carvalho F, Flanagan RJ. Haematological toxicity of clozapine and some other drugs used in psychiatry. Hum Psychopharmacol 2011;26:112-119.

2. Duggal HS, Gates C, Pathak PC. Olanzapine-induced neutropenia: mechanism and treatment. J Clin Psychopharmacol 2004;24:234-235.

3. Cowan C, Oakley C. Leukopenia and neutropenia induced by quetiapine. Prog Neuropsychopharmacol Biol Psychiatry 2007;31:292-294.

4. Sluys M, Guzelcan Y, Casteelen G, de Haan L. Risperidone-induced leucopenia and neutropenia: a case report. Eur Psychiatry 2004;19:117.

5. Yalcin DO, Goka E, Aydemir MC, Kisa C. Is aripiprazole the only choice of treatment of the patients who developed anti-psychotic agents-induced leucopenia and neutropenia? A case report. J Psychopharmacol 2008;22:333-335.

6. Vesta KS, Medina PJ. Valproic acid-induced neutropenia. Ann Pharmacother 2003;37:819-821.

7. Spina E, D’Arrigo C, Santoro V, Muscatello MR, Pandolfo G, Zoccali R, et al. Effect of valproate on olanzapine plasma concentrations in patients with bipolar or schizoaffective disorder. Ther Drug Monit 2009; 31:758-763.

8. Aichhorn W, Marksteiner J, Walch T, Zernig G, Saria A, Kemmler G. Influence of age, gender, body weight and valproate comedication on quetiapine plasma concentrations. Int Clin Psychopharmacol 2006;21: 81-85.

9. Nair P, Lippmann S. Is leucopenia associated with divalproex and/or quetiapine. Psychosomatics 2005;46:188-189.

10. Qureshi S, Rubin E. Risperidone- and aripiprazole-induced leukopenia: a case report. Prim Care Companion J Clin Psychiatry 2008;10:482483.

11. Thangadurai P, Jyothi KS, Gopalakrishnan R, Kuruvilla A, Jacob KS. 
Reversible neutropenia with olanzapine following clozapine-induced neutropenia. Am J Psychiatry 2006;163:1298.

12. Godleski LS, Sernyak MJ. Agranulocytosis after addition of risperidone to clozapine treatment. Am J Psychiatry 1996;153:735-736.

13. Montgomery J. Ziprasidone-related agranulocytosis following olanzapine-induced neutropenia. Gen Hosp Psychiatry 2006;28:83-85.

14. Lander M, Bastiampillai T. Neutropenia associated with quetiapine, olanzapine, and aripiprazole. Aust N Z J Psychiatry 2011;45:89.

15. Flanagan RJ, Dunk L. Haematological toxicity of drugs used in psychi- atry. Hum Psychopharmacol 2008;23(Suppl 1):27-41.

16. Jagadheesan K, Mehrtens J. Prolongation of clozapine-induced neutropenia with olanzapine. Aust N Z J Psychiatry 2007;41:192.

17. Sikora A, Adamus J, Marcinek A. Disproportionation of clozapine radical: a link between one-electron oxidation of clozapine and formation of its nitrenium cation. Chem Res Toxicol 2007;20:1093-1098.

18. Lahdelma L, Oja S, Korhonen M, Andersson LC. Clozapine is cytotoxic to primary cultures of human bone marrow mesenchymal stromal cells. J Clin Psychopharmacol 2010;30:461-463. 